\title{
Anticipatory reconfiguration elicited by fully and partially informative cues that validly predict a switch in task
}

\author{
Frini Karayanidis, Elise L. Mansfield, Kasey L. Galloway, Janette L. SMith, \\ Alexander Provost, and Andrew Heathcote \\ University of Newcastle, Callaghan, New South Wales, Australia
}

\begin{abstract}
Task-switching studies show no behavioral benefit of partially informative cues. However, ERP evidence of an early cue-locked positivity elicited by both fully and partially informative cues suggests that both cues trigger an anticipatory component of task set reconfiguration (Nicholson, Karayanidis, Davies, \& Michie, 2006). We examined this apparent discrepancy using a cued-trials task-switching paradigm with three tasks. The ERP finding of an early cue-locked positivity was replicated for both switch-to cues, which validly predicted an upcoming switch trial and specified the new task set, and switch-away cues, which validly predicted an upcoming switch trial but not the new task set. This component was not elicited by a noninformative cue that did not specify whether the task would switch or repeat. Switch-away cues resulted in more accurate but not faster responding than did noninformative cues. Modeling of decision processes confirmed a speed-accuracy trade-off between these conditions and a preparation benefit for both switch-to and switch-away cues. These results indicate that both fully and partially informative cues elicit an early anticipatory component of task set reconfiguration that is reflected in the early cue-locked positivity. We argue that the pattern of results is most consistent with a task set inhibition account of this early anticipatory component of task set reconfiguration.
\end{abstract}

Task-switching paradigms require rapid alternation between two or more task sets defined on the basis of distinct or partially overlapping target features. Typically, these paradigms produce switch costs - longer reaction times (RTs) and more errors when tasks are switched as compared with when tasks are repeated (e.g., Rogers \& Monsell, 1995). In cued-trial paradigms, increasing the cue-target interval reduces RT switch cost, but a significant residual switch cost remains even with long preparation intervals (Meiran, Chorev, \& Sapir, 2000). Recent behavioral (e.g., Arrington, Logan, \& Schneider, 2007) and electrophysiological (e.g., Karayanidis, Coltheart, Michie, \& Murphy, 2003) studies support multicomponent models of task switching, with switch cost reflecting both active control processes (e.g., task set reconfiguration; Rogers \& Monsell) and passive target-driven processes (e.g., stimulus-response [S-R] priming; Wylie \& Allport, 2000).

Although there is evidence of a role for inhibition in task switching, it is unclear at what stage an inhibitory mechanism may be activated and whether it is a top-down process or a bottom-up process. Mayr and Keele (2000) argued that a longer RT on the third trial of an ABA sequence, as compared with a CBA sequence, supports an inhibitory control process, albeit a rather low-level one, since inhibition was not overcome with increasing preparation. Koch and colleagues argued that this inhibition is a by-product of response activation, since studies have shown no backward inhibition (Schuch \& Koch, 2003) or RT switch cost (Koch \& Philipp, 2005) following no-go trials that require task set preparation but no response execution. Driesbach, Haider, and Kluwe (2002) compared subjective expectancy for partially informative cues, which signal an impending switch trial without identifying which specific task to prepare, and fully informative cues, which indicate which task to switch to. Unlike fully informative cues, partially informative cues did not produce subjective expectancy effects. Thus, knowledge that the task would change without specification of which task would be performed did not produce the differential response benefit that would be expected if inhibition of the previously active task set was required to switch tasks (see also Hubner, Dreisbach, Haider, \& Kluwe, 2003).

In contrast, Nicholson, Karayanidis, Davies, and Michie (2006) found event-related potential (ERP) evidence consistent with task set inhibition during the cue-target interval. ERPs are systematic fluctuations in brain electrical activity that are extracted from the electroencephalogram (EEG), using signal-averaging techniques (Andreassi, 2000), and have been shown to provide a high temporal resolution window into the processes underlying task switching. In particular, ERP waveforms time-locked to cue onset consistently show a larger parietal positivity for 
switch, as compared with repeat, trials (e.g., Kieffaber \& Hetrick, 2005; Miniussi, Marzi, \& Nobre, 2005; Nicholson, Karayanidis, Bumak, Poboka, \& Michie, 2006; Nicholson, Karayanidis, Poboka, Heathcote, \& Michie, 2005; Rushworth, Passingham, \& Nobre, 2005). This differential switch positivity (D-Pos) emerges as early as $200 \mathrm{msec}$ postcue and, with long preparation intervals, peaks prior to target onset. After target onset, ERPs for switch trials show a negative shift, relative to repeat trials, that emerges after $150 \mathrm{msec}$ and extends more than $800 \mathrm{msec}$ after target onset. D-Pos has been mapped to processes associated with task set reconfiguration during the cue-target interval, whereas the switch negativity has been mapped to target-dependent processes that cause residual switch cost (Karayanidis et al., 2003; Nicholson, Karayanidis, Bumak, et al., 2006; Nicholson et al., 2005).

Nicholson, Karayanidis, Davies, and Michie's (2006) ERP evidence for task set inhibition came from a cued- trial task-switching paradigm in which participants randomly alternated between three tasks. As is usual in task-switching paradigms, different cues signaled task repetition (repeat cue) or a switch to a specified task (switch-to cue). A third, partially informative cue signaled only that the task would change (switch-away cue), with the actual task to be performed being specified only upon target onset (see Figure 1). An early cue-locked differential positivity (D-Pos1) was found for both switchaway and switch-to trials, relative to repeat trials (see Figure 3B in Nicholson, Karayanidis, Davies, and Michie, 2006). Switch-to trials also showed a second differential positivity that occurred later within the cue-target interval (D-Pos2), whereas for switch-away trials, this component occurred after target onset. After target onset, both types of switch trials showed a differential negativity relative to repeat trials, but this was delayed until after D-Pos2 on switch-away trials.
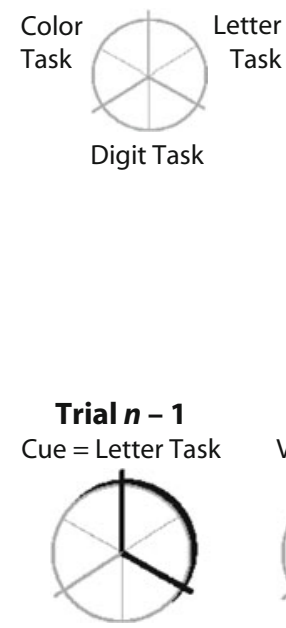

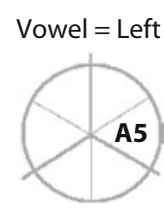

Trial $n$

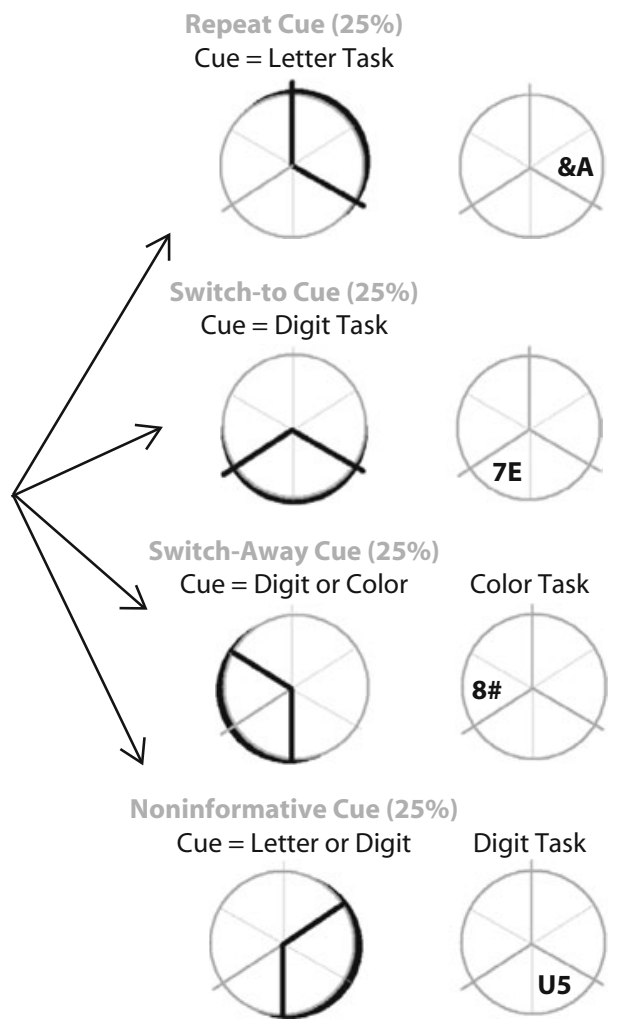

\begin{tabular}{cll} 
Example of S-R Mapping & \multicolumn{1}{c}{ Left-Hand Response } & Right-Hand Response \\
\hline Letter Task & Vowel (A, E, I, U) & Consonant $(\mathrm{G}, \mathrm{K}, \mathrm{M}, \mathrm{R})$ \\
Digit Task & Even $(2,4,6,8)$ & Odd $(3,5,7,9)$ \\
Color Task & Cold & Hot \\
& (dark green, light green, & (red, pink, orange, yellow) \\
& dark blue, turquoise) & \\
\hline
\end{tabular}

Figure 1. Top left: Example of task-position mapping. Middle: The four types of cues used to indicate the requirements of the next trial (repeat, switch-to, switch-away, and noninformative). Each cue type was presented on $25 \%$ of trials. Bottom: Stimulusresponse $(\mathrm{S}-\mathrm{R})$ mappings with the four possible stimuli associated with each response. These were counterbalanced across participants. 
Nicholson, Karayanidis, Davies, and Michie (2006) suggested that D-Pos2 reflects activation of the relevant task set, which can occur prior to target onset for switch-to trials, but only after target onset for switch-away trials. Since both switch-to and switch-away trials indicate that the previously active task set will not be repeated, the D-Pos1 component, which was common to both of these trial types, was interpreted as reflecting inhibition of the now irrelevant task set. However, D-Pos1 might also be attributed to differences in cue processing between repeat and both types of switch trials. In particular, both switch-to and switch-away trials involved a physical cue change between trials, which may have resulted in greater cue processing being required than for the cue on a repeat trial, where cue processing may have been primed by cue repetition.

Nicholson, Karayanidis, Davies, and Michie (2006) found that switch cost was larger on switch-away, as compared with switch-to, trials and that increasing the cue-target interval reduced RT switch cost for switch-to, but not switch-away, trials. The length of the cue-target interval may have had no effect on switch-away trials because task set inhibition was complete before the target appeared, even at the short cue-stimulus interval $(200 \mathrm{msec})$. However, it is also possible that there was no cue-target interval effect on switch-away trials simply because participants did not make any use of the switch-away cue. The equivalence of early ERP waveforms (D-Pos1) for switch-to and switch-away could then be attributed to participants' undertaking the same cue-encoding processes in both conditions. If a switch-away cue does allow partial preparation, it should cause a reduction in switch cost, even though this reduction would be less than that for switch-to cues. However, the behavioral benefits of the switch-away cue could not be established by Nicholson, Karayanidis, Davies, and Michie since their design did not have a baseline condition.

\section{EXPERIMENT 1}

The present study addressed these issues, using a design identical to that in Nicholson, Karayanidis, Davies, and Michie (2006), with the exception that an extra, noninformative cue type was included. This cue signaled that the following trial might require a repeat or a switch in task (see Figure 1). Like switch-to and switch-away cues, noninformative cues involved a physical shift in cue position. However, unlike switch-to and switch-away cues, noninformative cues did not indicate that the previously active task set would be irrelevant on the next trial. In fact, they indicated that there was a $50 \%$ chance that the task would be repeated. Although inhibition of the previously active task set is an efficient strategy for switch-to and switch-away cues, this is not the case for noninformative cues. Hence, if D-Pos1 represents processes associated with inhibition of the previously active but now irrelevant task set, it should occur for switch-to and switch-away cues, but not for noninformative cues. Alternatively, if D-Pos1 represents processing of the change in cue position, it should occur for switch-to, switch-away, and noninformative cues.
The noninformative cue condition also acted as a baseline that allowed us to investigate whether switch-away cues provide some behavioral benefit (i.e., reduce switch cost) by allowing partial preparation for a task switch. Note that noninformative and switch-away cues were equally informative about which task would occur next. That is, both of these cue types ruled out exactly one of the three possible tasks. Hence, a comparison of performance for these cue types controlled for task uncertainty and, specifically, tested for benefits related to knowing that the previous task would not be repeated.

\section{Method}

Participants. Twenty-three undergraduate students (18 female, 5 male) with a mean age of 21.3 years $(S D=3.51)$ were recruited from an introductory psychology course and participated for course credit.

Stimuli and Tasks. The paradigm was identical to that used by Nicholson, Karayanidis, Davies, and Michie (2006) with the exception of the additional noninformative cue. Briefly, the participants viewed a circle ( $5^{\circ}$ of visual angle) divided into six wedges, with pairs of adjacent wedges grouped by thicker lines demarcating three task sections: digit, letter, and color (see Figure 1, top). Each target was a pair of characters consisting of combinations of a letter, a digit, or a nonalphanumeric symbol and was presented either in gray or in color. Each target (e.g., A4) consisted of three dimensions (see Figure 1, bottom): one relevant to the currently cued task (e.g., a letter mapped to a left-hand response), one selected randomly from one of the two alternative tasks and incongruently mapped with the relevant task (e.g., a digit mapped to a right-hand response), and one that was neutral (e.g., gray, not mapped to any response). The same target could not appear on two successive trials. Response-target interval was $1,400 \mathrm{msec}$ and included a $1,000 \mathrm{msec}$ cue-target interval.

Four cue types (i.e., repeat, switch-to, switch-away, and noninformative) were defined by cue location and were presented with equal probability in a pseudorandom sequence so that the same cue was not repeated on more than three consecutive trials. Noninformative cues resulted equiprobably in a switch or a repeat trial, which was defined by the location of the target, thereby resulting in five trial types (i.e., repeat, switch-to, switch-away, noninformative switch, and noninformative repeat). The target always appeared in one of the two segments highlighted by the cue.

Procedure. All the participants attended two sessions scheduled 7-14 days apart. The first session included task training and practice ( 732 trials on each task alone and switching between tasks). The second session included further practice (another 732 trials), followed by the behavioral and EEG testing session. The testing session consisted of nine runs of 96 trials each. The participants were encouraged to respond as quickly and accurately as possible. Auditory feedback was provided after an incorrect response, and behavioral feedback (mean RT and percentage correct) was displayed at the end of each run. EEG was continuously sampled at $2048 \mathrm{~Hz} /$ channel, reference free, from 64 scalp electrodes, the mastoids, and the nose, using a Biosemi ActiView II system. A vertical electrooculogram (EOG) was recorded from the supraorbital and infraorbital ridges of the left eye, and a horizontal EOG from the outer canthi of each eye.

Data analysis. The first five trials of every run, trials associated with an incorrect response, trials immediately following an incorrect response, and trials on which RTs were shorter than $200 \mathrm{msec}$ $(0.005 \%)$ or longer than three standard deviations above the participant's mean RT (1.7\%) were excluded. A 3 (task: letter, digit, color) $\times 5$ (trial: repeat, switch-to, switch-away, noninformative repeat, and noninformative switch) repeated measures ANOVA was performed. Critical values were adjusted using the GreenhouseGeisser correction to avoid violating the assumption of sphericity (Vasey \& Thayer, 1987), and simple comparisons for trial were cor- 
rected with familywise error rate adjusted at $\alpha=.01$ (unless otherwise reported). For behavioral data, we compared repeat trials with each of switch-to, switch-away, and noninformative repeat trials, and switch-away trials with each of switch-to and noninformative switch trials. Task did not interact with trial type for either RT $(F=1.64)$ or error rate $(F=2.43)$, so all behavioral and ERP analyses were averaged over task.

EEG data were analyzed using Brain Electrical Source Analysis (BESA v5.1). Scalp electrodes were rereferenced offline to linked mastoids, and EOG artifact correction was applied using a regression algorithm (Ille, Berg, \& Scherg, 2002). Cue- and target-locked EEG epochs were extracted from $300 \mathrm{msec}$ before to $1,200 \mathrm{msec}$ after each cue and target (200-msec preevent baseline), and epochs with an artifact exceeding a $100-\mu \mathrm{V}$ threshold were rejected. Averaged waveforms were created for each cue and target type, averaged over response hand and task. Both cue-locked and target-locked individual ERP waveforms included a mean of 130-140 trials, except for target-locked noninformative switch and repeat trials, which included half that number. Target-locked data from 2 participants were excluded because there were fewer than 40 epochs contributing to one of the noninformative trial types. Therefore, cue-locked data are reported from 23 participants, and target-locked data are from 21 participants.

Difference waveforms were calculated by subtracting the repeat waveform from each of the remaining waveforms and were visually inspected to determine time windows and scalp topography of maximal differentiation between cue types. For cue-locked waveforms, two mean amplitude windows were defined on the basis of the positivity for switch-to relative to repeat waveforms $(250-400 \mathrm{msec}$, $450-700 \mathrm{msec}$ ) and were analyzed at the parieto-occipital midline site (POz), using a one-way ANOVA with four levels of cue type. We compared repeat cues with each of the other three cues and switchaway with switch-to and noninformative cues. For target-locked waveforms, two mean amplitude windows were used to define an early positivity that emerged around the peak of the $\mathrm{P} 2$ and a second, later positivity around the latency of the N2 (180-250 and 300$370 \mathrm{msec}$, respectively) and were analyzed at F4, where the effects of trial were maximal. A third window $(420-550 \mathrm{msec})$ that targeted the negativity for switch-to relative to repeat trials was analyzed at $\mathrm{Cz}$. Four contrasts were defined comparing repeat trials with each of the other three trial types. Where significant trial type differences emerged at these scalp sites, the scalp distribution of these differences was analyzed using paired-samples $t$ tests at each electrode and are displayed as head maps in Figures 2 and 3.

\section{Results}

Because Nicholson, Karayanidis, Davies, and Michie's (2006) argument that task set reconfiguration involves task set inhibition as well as task set activation was based on ERP data, we will discuss first the ERP findings in order to establish replication of the original finding and present the outcomes for the noninformative cue. Figures 2 and 3 show average cue-locked and target-locked waveforms, respectively. Figure 4 shows average behavioral and ERP estimates and the results of associated inferential tests.

Cue-locked waveforms. Cue-locked waveforms showed a sustained positivity over $100-800 \mathrm{msec}$ for all trial types (Figure 2A, left). The main effect of cue was significant at $\mathrm{POz}$ for both early and late positivities $[F(3,66)=18.10, p<.001$, and $F(3,66)=8.45, p<$ .001 , respectively]. Difference waveforms were derived between each cue type and the repeat waveform (Figure $2 \mathrm{~A}$, right). A large broad positivity was evident over $150-800 \mathrm{msec}$ in the switch-to difference waveform. This was also evident in the switch-away difference waveform, but dissipated by $400 \mathrm{msec}$. Noninformative cues did not show any positivity relative to repeat cues.

The early positivity was significantly larger for both switch-to and switch-away cues as compared with repeat cues $[F(1,22)=39.30, p<.001$, and $F(1,22)=31.68$, $p<.001$, respectively; see Figure 4B]. This differential positivity for switch-to and switch-away relative to repeat cues emerged at central sites but was stronger at parietal and occipital sites and was also reflected at frontopolar locations (Figure 2B). Importantly, this early positivity was also larger for switch-away cues, as compared with noninformative cues $[F(1,22)=17.89, p<.001]$, across most parietal-occipital sites (Figure $2 \mathrm{~B}$ ) and did not differ in amplitude between repeat and noninformative cues at any site. The later positivity was larger for switch-to cues than for both repeat cues and switch-away cues at $\mathrm{POz}[F(1,22)=9.31, p=.006$, and $F(1,22)=12.65$, $p=.002$, respectively; see Figure 4B], an effect that was distributed over the parietal-occipital scalp (Figure 2B). There was no difference between the other cue types in this latency range.

Target-locked waveforms. Target-locked waveforms showed an early N1 and large fronto-central P2, followed by an N2 and LPC complex (Figure 3A, left). Switch-to minus repeat difference waveforms showed a broad negative shift spreading over $200-800 \mathrm{msec}$ after target onset that was largest at $\mathrm{Cz}$ (Figure 3A, right). All other difference waveforms showed a right frontally maximal positivity over $200-400 \mathrm{msec}$, followed by a broad centrally maximal negativity.

Target-locked difference waveforms for switch-away, noninformative repeat, and noninformative switch targets showed two positive peaks: one within the latency range of the frontal P2 (180-250 msec) and the other around $100 \mathrm{msec}$ later (300-370 msec; Figure 3A, right). Both windows showed a significant main effect of trial type at $\mathrm{F} 4[F(4,80)=3.30, p=.043, \varepsilon=.538$, and $F(4,80)=$ $7.37, p<.001$, respectively]. The early positivity (180$250 \mathrm{msec}$; Figure 4C) was larger for both switch-away and noninformative repeat targets, as compared with repeat targets $[F(1,20)=8.45, p=.009$, and $F(1,20)=11.85$, $p=.003$, respectively]. This early target-locked differential positivity was more widespread over frontocentral sites for noninformative repeat cues but was fairly localized over the right frontal scalp for switch-away cues (Figure $3 \mathrm{~B})$. The later positivity $(300-370 \mathrm{msec})$ was larger for both noninformative repeat and noninformative switch targets, as compared with repeat targets $[F(1,20)=10.77$, $p=.004$, and $F(1,20)=9.03, p=.007$, respectively; see Figure 4C], over both right frontocentral and left centroparietal sites (Figure 3B). This positivity was again evident for switch-away cues but was only marginally significant over the right frontal scalp $[F(1,20)=4.77, p=.041]$.

Mean amplitude over 420-550 msec in the target-locked waveforms produced a significant main effect of trial at $\mathrm{Cz}[F(4,80)=6.38, p=.002$; see Figure $3 \mathrm{~A}]$, reflecting a significant negative deflection for all trial types, relative to repeat targets [see Figure 4C; switch-to, $F(1,20)=$ $16.65, p=.001$; switch-away, $F(1,20)=19.45, p<.001$; 
A

\section{Cue-Locked ERP Waveforms}

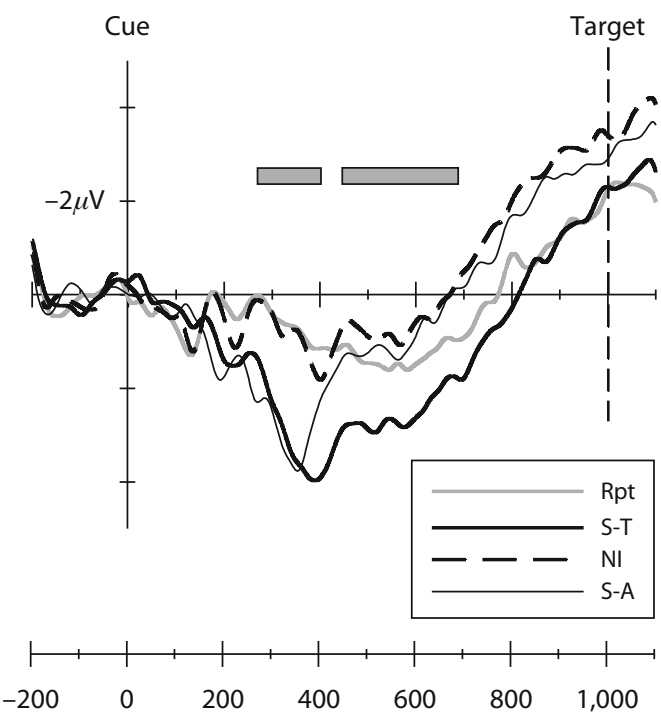

Difference Waveforms
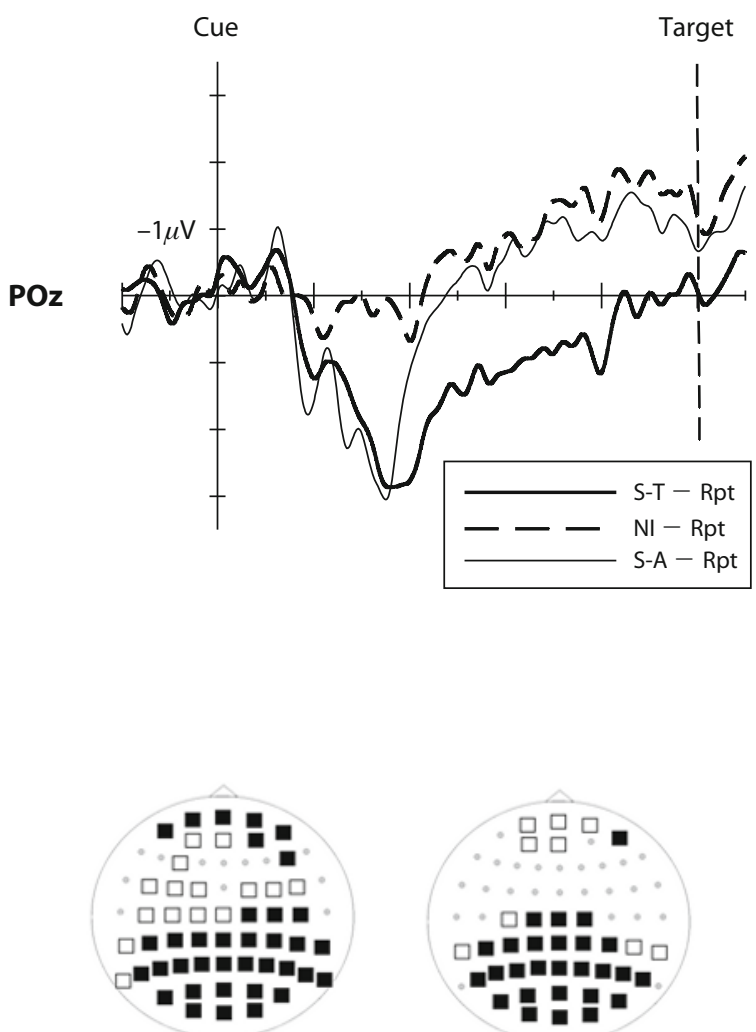

S-A vs. Rpt

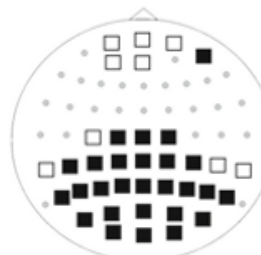

S-A vs. NI

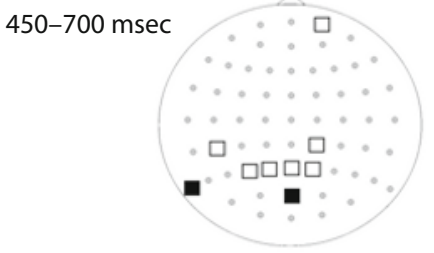

S-T vs. Rpt

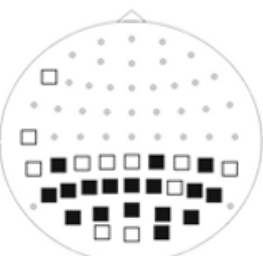

S-T vs. S-A

Figure 2. (A) Cue-locked ERP and difference waveforms for each trial type at POz. Gray bars indicate the mean amplitude windows used in the analyses. (B) Head maps showing sites of significant deviation between different trial types over 250-400 msec and 450-700 msec. Open squares, $\alpha=.05$; filled squares, $\alpha=.01$. Over 250-400 msec, the switch-to (S-T) versus repeat (Rpt) contrast was most significant over parieto-occipital sites $\left(p=2.6 \times 10^{-6}\right.$ to .005$)$. The switch-away (S-A) versus Rpt contrast was most significant over frontal and parieto-occipital electrodes $\left(p=2.5 \times 10^{-8}\right.$ to .01). The noninformative (NI) versus S-A contrast was most significant over parieto-occipital electrodes $\left(p=5.1 \times 10^{-5}\right.$ to .01). Over 450-700 msec, the $S-T$ versus Rpt contrast was most significant at $P O z(p=.009)$. The $S$-T versus $S$-A contrast was most significant at parieto-occipital sites $(p=.0009$ to .01$)$.

noninformative switch, $F(1,20)=8.37, p=.009$; noninformative repeat, $F(1,20)=10.03, p=.005]$. This posttarget switch negativity showed a broad scalp distribution for switch-to and switch-away targets, especially over centroparietal sites (Figure 3B), whereas the effect was restricted over the frontocentral midline for both noninformative switch and noninformative repeat targets.

Accuracy and mean RT. Mean RT showed a significant effect of trial type $[F(4,88)=38.62, p<.001$, $\varepsilon=.320$; see Figure 4A]. Responses for repeat trials were significantly faster than those for noninformative repeat $[F(1,22)=61.49, p<.001]$, switch-to $[F(1,22)=32.29$, $p<.001]$, and switch-away $[F(1,22)=51.59, p<.001]$ trials. RT for switch-away trials was longer than for switchto trials $[F(1,22)=37.36, p<.001]$, but was not significantly shorter than that for noninformative switch trials.

Although the error rate was quite low $(2.8 \%-5.5 \%$; see Figure 4A), the main effect of trial type was significant $[F(4,88)=10.76, p<.001, \varepsilon=.673]$. Repeat trials produced fewer errors than did all other trial types [switch-to, 
A Target-Locked ERP Waveforms

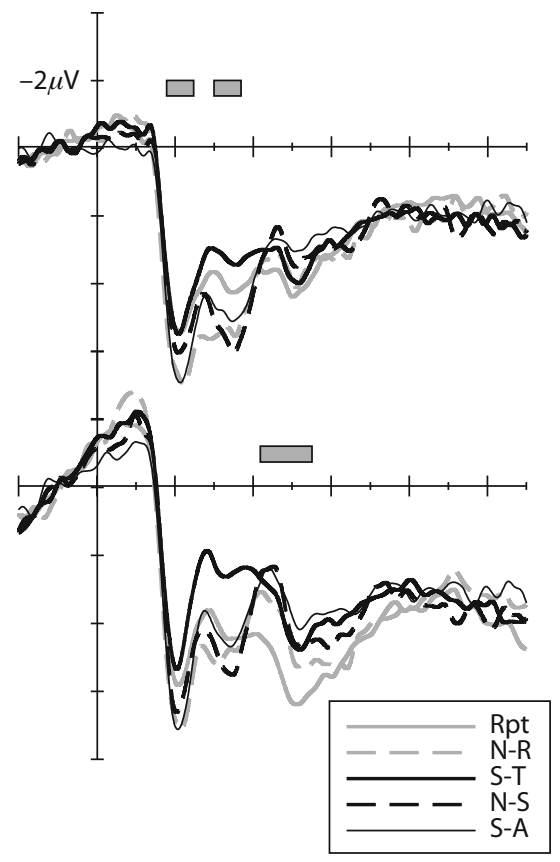

\section{Difference Waveforms}

F4

$\mathrm{Cz}$
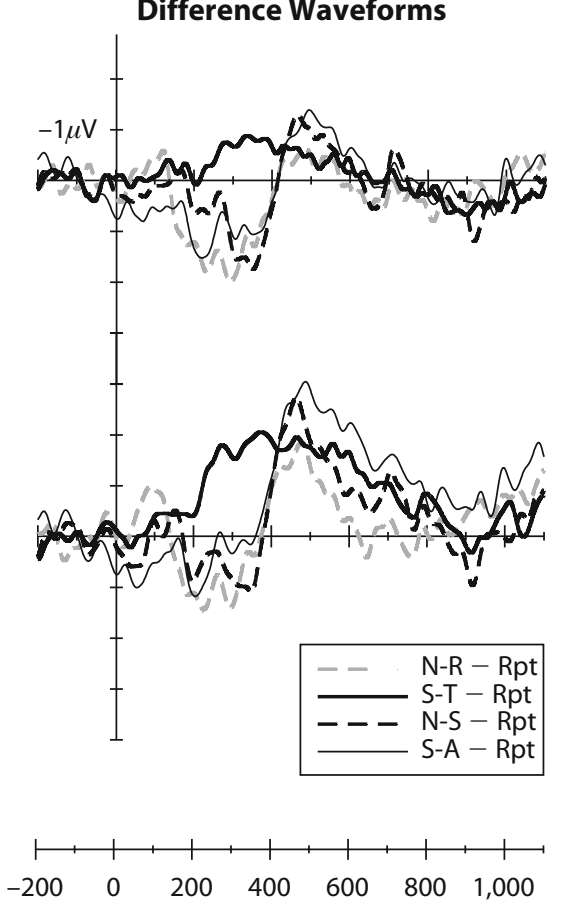

B

$180-250 \mathrm{msec}$

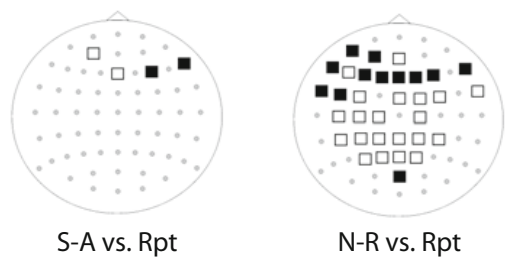

300-370 msec
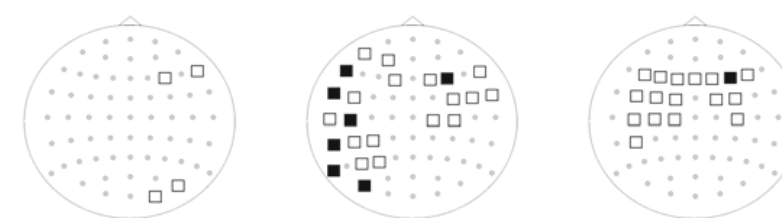

S-Aw vs. Rpt

N-R vs. Rpt

$\mathrm{N}-\mathrm{S}$ vs. Rpt

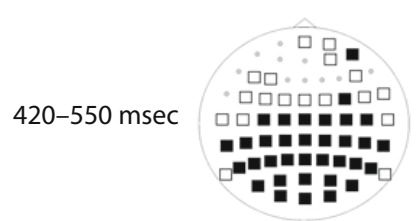

S-T vs. Rpt

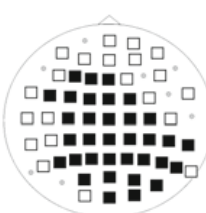

S-A vs. Rpt

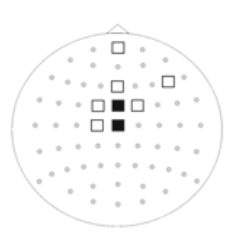

N-R vs. Rpt

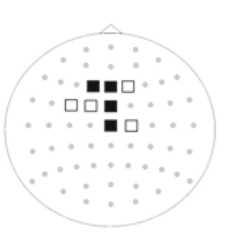

N-S vs. Rpt

Figure 3. (A) Target-locked ERP and difference waveforms at F4 and Cz. Gray bars indicate the respective mean amplitude windows used in analysis. (B) Head maps showing sites of significant positive deviation relative to repeat (Rpt) trials over 180-250, 300-370, and 420-550 msec. Open squares, $\alpha=.05$; filled squares, $\alpha=.01$. Over 180-250 msec, the switch-away (S-A) versus Rpt contrast was most significant at F4 $(p=.009)$. The noninformative repeat $(N-R)$ versus Rpt contrast was most significant over frontal sites $(p=.002$ to .01$)$. Over 300-370 msec, the S-A versus Rpt contrast was most significant at F8 $(p=.02)$. The N-R versus Rpt contrast was most significant over left centro-parietal sites $(p=.0009$ to .01$)$. The noninformative switch (N-S) versus Rpt contrast was most significant at F4 ( $p=.007)$. Over $420-550$ msec, the switch-to (S-T) versus Rpt contrast and the S-A versus Rpt contrast were most significant over centroparieto-occipital electrodes $(p=.0001$ to .009 and $p=.0003$ to .009 , respectively). The $\mathrm{N}-\mathrm{R}$ versus Rpt contrast was most significant at $\mathrm{Cz}(p=.005)$, and the $\mathrm{N}-\mathrm{S}$ versus Rpt contrast was most significant over fronto-central sites $(p=.004$ to .009$)$. 
A

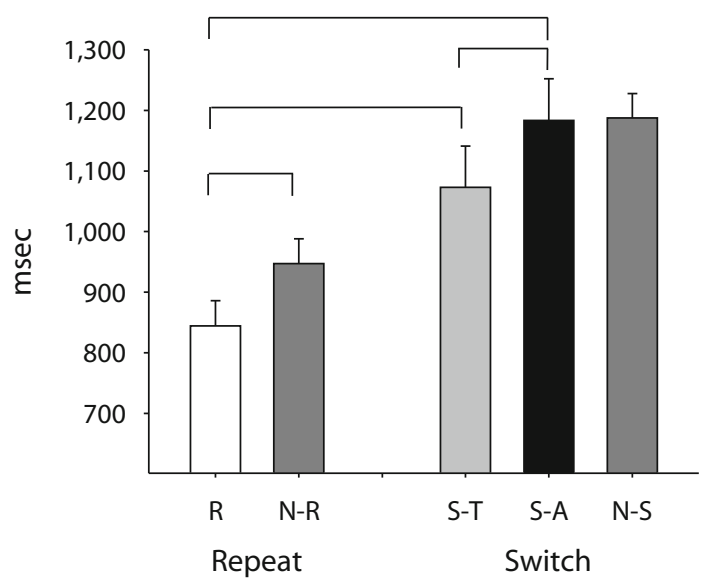

Error Rate

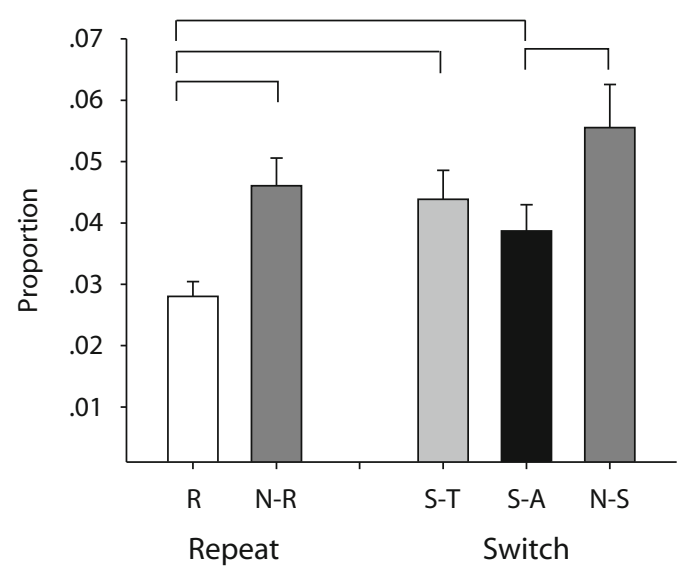

B

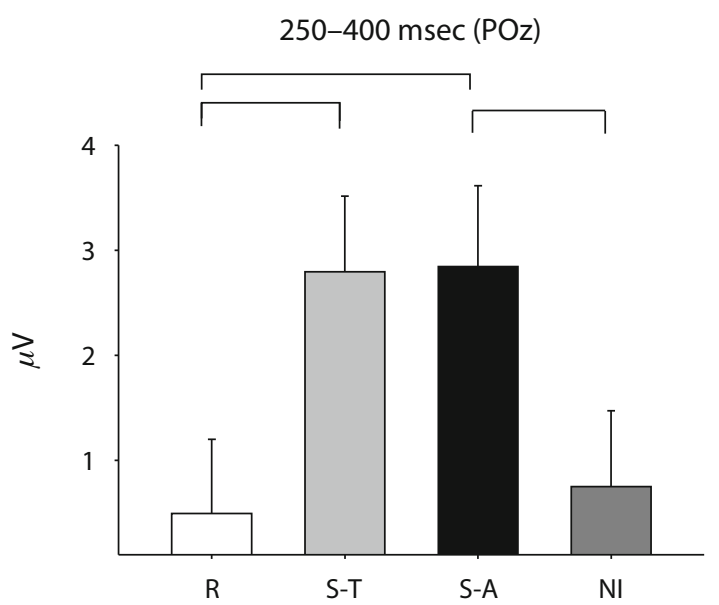

\section{Cue-Locked Mean Amplitude}

450-700 msec (POz)

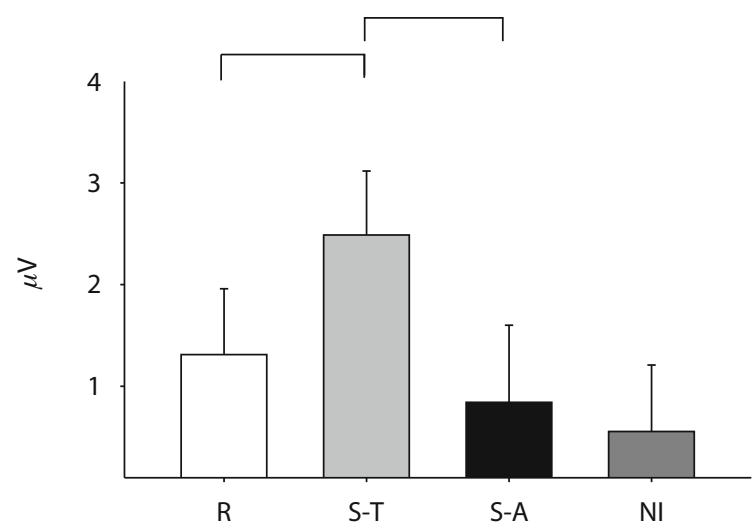

C 180-250 msec (F4)

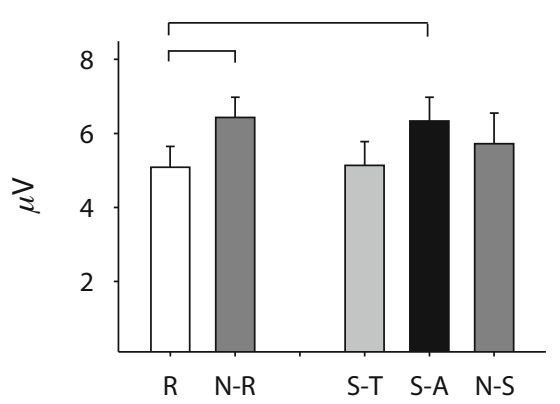

Target-Locked Mean Amplitude

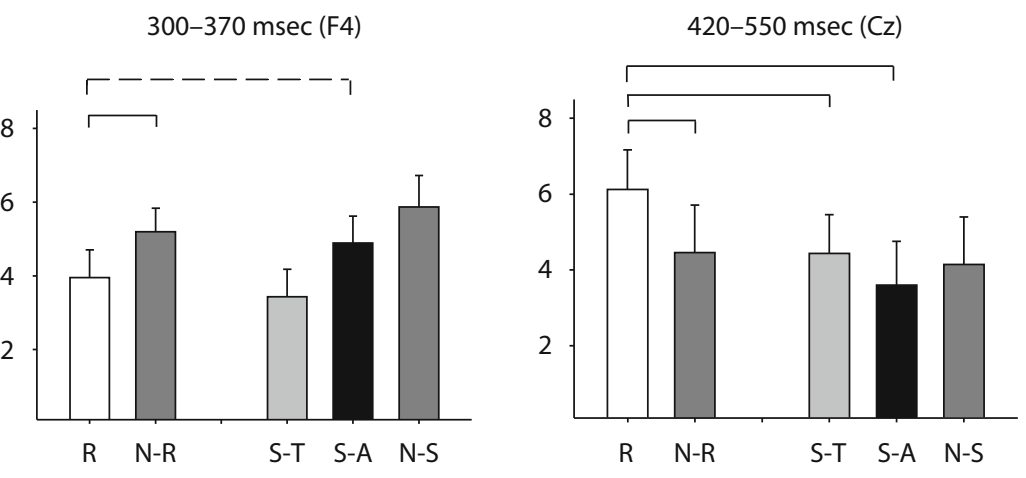

Figure 4. (A) Mean RT and error proportion for each trial type. (B) Cue-locked ERPs: mean amplitude over $250-400$ and $450-$ 700 msec at POz. (C) Target-locked ERPs: mean amplitude over 180-250 and 300-370 msec at F4 (left, middle) and over 420-550 msec at $\mathrm{Cz}$ (right). R, repeat; N-R, noninformative repeat; S-T, switch-to; S-A, switch-away; N-S, noninformative switch. Significant differences between conditions are shown by solid lines at $p<.01$ and broken lines at $p<.05$. 
$F(1,22)=16.87, p<.001$; switch away, $F(1,22)=9.45, p=$ .006 ; noninformative repeat, $F(1,22)=21.27, p<.001]$. Error rates were also higher for noninformative switch than for switch-away trials $[F(1,22)=9.53, p=.005]$.

We examined whether the amplitude of the early cuelocked positivity was associated with improved behavioral performance, using one-tailed Pearson correlations for switch-to and switch-away cues, which showed a clear and measurable D-Pos1. Larger positivity was associated with a shorter RT for switch-to trials $(r=-.691, p<.001, n=$ $23)$ and less strongly for switch-away trials $(r=-.367, p<$ $.05, n=23$ ) but showed no relationship with error rate.

\section{Discussion}

The ERP data replicated Nicholson, Karayanidis, Davies, and Michie's (2006) finding of a posterior cue-locked D-Pos1 for both switch-to and switch-away cues, followed by a D-Pos 2 that was cue-locked for switch-to trials and target-locked for switch-away trials. Both switch-to and switch-away trials elicited a large posttarget switch negativity, as compared with repeat trials, and the onset of this negativity was delayed until after resolution of the earlier positivity for switch-away trials, again suggesting that it reflects target-triggered processes such as completion of task set reconfiguration or $\mathrm{S}-\mathrm{R}$ priming.

Notably, within the cue-target interval, noninformative cues showed no evidence of any differential switch positivity, relative to repeat cues. However, after the onset of the target that defined the currently active task set, both noninformative repeat and noninformative switch trials showed a significant differential positivity, relative to repeat trials. The finding that, unlike switch-to and switch-away cues, noninformative cues did not elicit the D-Pos1 within the cue-target interval indicates that this component does not reflect processing of a change in the physical position of the cue. It could be argued that although noninformative cues involved some spatial displacement, the degree of displacement differed between cue types (i.e., $60^{\circ}$ for noninformative, $120^{\circ}$ for switch-to, and $180^{\circ}$ for switch-away cues). However, if the cue-locked positivity is affected by degree of cue displacement, there should be correspondence between the angular displacement of the cue and D-Pos1 amplitude (i.e., noninformative $<$ switch-to $<$ switch-away). This was not the case in the present data.

Therefore, the D-Pos1 component appears to reflect a process that is activated by cues that validly signal that the previously active task set will not be relevant to the next target and, consequently, that there will definitely be a switch in task on the next target (i.e., switch-to and switchaway cues), even when the cues do not specify which task will be relevant. Importantly, the process reflected by the D-Pos1 is not activated by cues that signal that the previously active task set may (i.e., noninformative cues) or will (repeat) be relevant to the next target. This finding supports the contention that partially informative cues trigger some anticipatory reconfiguration process.

Replicating Nicholson, Karayanidis, Davies, and Michie's (2006) finding, switch-away trials resulted in a longer RT than did switch-to trials. This indicates that the additional information regarding the identity of the upcoming task afforded by switch-to cues led to greater anticipatory reconfiguration than on switch-away cues. However, mean RT did not differ between switch-away and noninformative switch trials. This result appears to contradict the idea that participants use switch-away cues to partially prepare for a switch trial. If preparation is a time-consuming process, it should take longer to complete on noninformative switch trials than on the partially informative switch-away trials, and hence, mean RT should be less in the latter condition.

This argument fails to take account of the fact that the noninformative switch trials had a reliably higher error rate than did switch-away trials. The error difference raises the possibility that the participants used the information provided by switch-away cues to engage in a speed-accuracy trade-off. That is, because switch-away cues provided certainty that the upcoming trial would require a switch in task and, hence, would be more difficult and potentially error prone, the participants may have required a higher standard of evidence before making a decision, in order to reduce the possibility of making an error. If that were the case, the same higher standard of evidence would be expected to be applied on switch-to trials. Mean RT in the switch-to condition could still be less than that in the noninformative switch condition if the extra time required to make a decision using a higher standard of evidence on switch-to trials was less than the time saved by being able to complete reconfiguration in the cue-target interval. In the switch-away condition, in contrast, the lesser amount of time saved by partial reconfiguration could be canceled out by the extra time taken to make a decision, so that overall mean RTs in the switch-away and noninformative switch conditions would be equal.

Fortunately, as we will describe next, it is possible to directly test our speculation about speed-accuracy tradeoff differences between cue conditions. Speed-accuracy trade-off is a pervasive phenomenon in choice tasks ranging from simple stimulus categorization to recognition memory (for a summary, see Luce, 1986, pp. 237-245). It has been intensively studied, and it is now almost universally agreed that it can be explained in detail by evidence accumulation models. Evidence accumulation models of the decision process provide a detailed account of the mechanism by which speed-accuracy trade-offs are accomplished. They also predict that a speed-accuracy trade-off will have a quite specific effect on aspects of the RT distribution, such as RT variance, which are neglected by an analysis of mean RT alone. Hence, by fitting an evidence accumulation model to our data, we are able to provide a rigorous test of whether the lack of a mean RT difference between noninformative and switch-away trials is a by-product of speed-accuracy trade-off.

\section{EVIDENCE ACCUMULATION MODEL ANALYSIS}

Evidence accumulation models fractionate mean RT within two-choice response tasks into two independent components: decision time and nondecision time. Decision time includes processes directly involved in choos- 
ing a response to the current stimulus - that is, stimulus categorization and response selection. Nondecision time includes the time to complete processes that do not directly contribute to the decision, typically including processes such as stimulus encoding and response activation/ execution. Evidence accumulation models assume that a decision is reached by accumulating (i.e., repeatedly sampling and combining) stimulus information about a choice until the evidence favoring one choice exceeds the evidence favoring other choices by a criterion amount. Decision time, therefore, is determined by the conservativeness of the evidence criterion and the rate of evidence accumulation. A speed-accuracy trade-off occurs when participants differ between conditions in conservativeness (i.e., maintain a different evidence criterion).

Wagenmakers, van der Maas, and Grasman (2007) advocated the use of parameter estimates from a particular type of evidence accumulation model, a diffusion model, to account for speed-accuracy trade-off. Their EZ diffusion method estimates three parameters. The evidence accumulation, or drift rate $(v)$ and the evidence criterion (a) parameters together determine decision time $(d t)$. The remaining portion of the RT that is due to nondecision processes is determined by the Ter parameter. We applied a more recent development of this approach, the EZ2 method (Grasman, Wagenmakers, \& van der Maas, 2009), which also estimates a decision bias parameter, although this parameter is not of substantive interest in the present application.

Within task-switching paradigms, when reconfiguration is completed before target onset (i.e., anticipatory reconfiguration with predictable switch cues and long cuetarget interval), there is no effect of reconfiguration on $\mathrm{RT}$, and any residual RT switch cost is assumed to reflect posttarget processes related to S-R priming. However, if reconfiguration is not completed before target onset (i.e., very short cue-target interval and/or unpredictable switch trials), RT will increase by the amount of time required to complete reconfiguration, since the initiation of decision processing will be delayed. Such delays will increase estimates of the nondecision time ( $\mathrm{Ter}$ ) parameter. In our paradigm, switch-to trials allow complete reconfiguration before target onset, and so there should be little or no contribution of reconfiguration to nondecision time. In contrast, on noninformative switch trials, reconfiguration should make a large contribution to nondecision time. If switch-away trials involve partial reconfiguration, nondecision time should be less in switch-away than in noninformative switch trials.

In summary, we predict that nondecision time should be shortest for switch-to trials, intermediate for switchaway trials, and longest for unprepared noninformative switch trials, since the amount of reconfiguration that can be completed in the cue-target interval decreases across these conditions. Predictions related to nondecision time for the repeat cue trials are less constrained, because the reconfiguration process itself may be primed in this condition. Generally, we would expect repeat trials to have a shorter nondecision time than all other trial types, because they require no reconfiguration or, at least, minimal re- configuration. Switch-to trials may be an exception, since the relatively long cue-target interval may have been sufficient to complete preparation to the same level as that on repeat trials.

Decision time is determined by criterion and drift rate; a longer decision time may result from a high response criterion, a lower drift rate, or a combination of both. Hence, if, as we suggested, participants use a more cautious (larger) evidence criterion in the switch-away than in the noninformative switch condition, a longer decision time would be predicted in the former condition. We argue that it is the fact that these two conditions have opposite effects on nondecision and decision time that can account for our finding of no difference between them in mean RT. Since both switch-to and switch-away cues certainly indicate that the next trial will be a switch, no difference in criterion or decision time is predicted between these conditions. However, we predict that switch-to trials will have a shorter mean RT because of their shorter nondecision time.

\section{Method}

Wagenmakers et al.'s (2007) EZ diffusion method estimates three separate parameters for each response to a task: the evidence accumulation, or drift, rate $(v)$; the evidence criterion $(a)$, which together determine mean decision time $(d t)$; and a parameter for the remaining portion of mean RT, nondecision time (Ter). These three parameters are estimated analytically on the basis of three aspects of the data for each response: accuracy and the mean and variance of RT for correct decisions. The EZ method assumes that decisions are unbiased, whereas the more recently developed EZ2 method (Grasman et al., 2009) does not need to make this assumption, since parameter estimates are obtained for the entire task, rather than for each response separately. These parameters are two drift rate and two nondecision time parameters (one for each response), the criterion for one of the responses ( $a$; the criterion for the other response is assumed to be zero without loss of generality), and the starting point for evidence accumulation $(z)$. These size parameters are estimated on the basis of six data points, accuracy, and the mean and variance of correct RT for each response.

Hence, as is the case for EZ, the number of parameters estimated equals the number of data points, but for EZ2, the equation relating the two cannot be solved analytically. However, the EZ2 equation implicitly defines a unique solution that can be easily and reliably found by numerical methods using programs provided by Grasman et al. (2009). Our use of EZ2 was not motivated so much by its affording an estimate of response bias (which we do not report, since there was no evidence of bias or differences in bias across conditions) as by the fact that it requires fewer assumptions and is, in our experience, more robust and efficient than EZ estimation, and because it corresponds more directly to the diffusion model's assumption that one evidence accumulation process is responsible for both choices.

In our experiment, mean RT showed a reliable difference between tasks and a reliable interaction between task and response hand. Since EZ2 analysis depends on variance estimates, and these can be distorted by pooling over conditions that differ in their mean, we applied the diffusion analysis to data broken down by task and response, as well as by trial type. This resulted in small sample sizes for correct responses (less than 20) for some conditions in some participants.

In order to make mean and variance estimates robust, we based them on fits of the ex-Gaussian distribution to correct RT deciles (Heathcote, Brown, \& Mewhort, 2002; see Wagenmakers, van der Maas, Dolan, \& Grasman, 2008, for a related approach to EZ estimation). We also based EZ2 estimates on the robust accuracy measure recommended by Snodgrass and Corwin (1988). In a few cases 


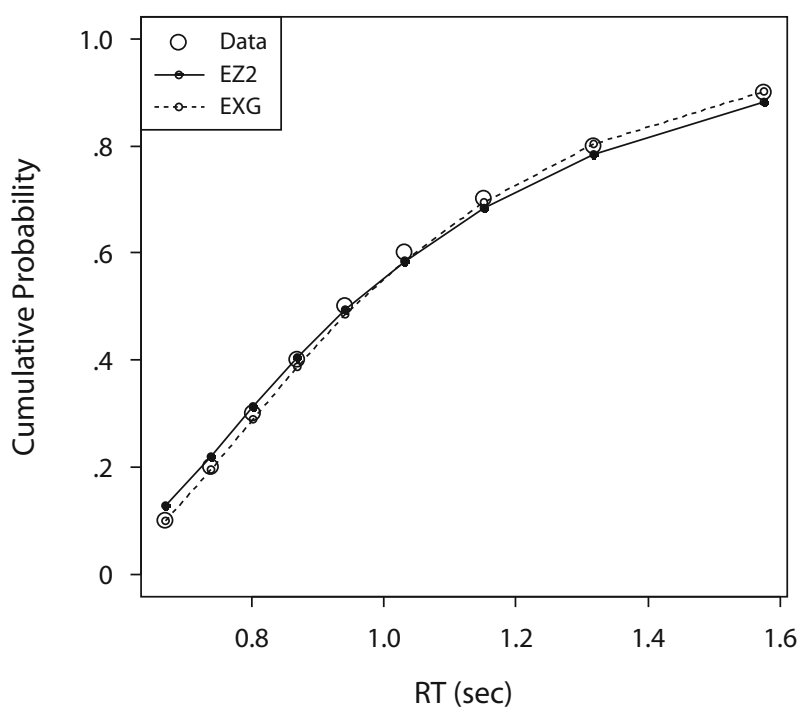

Figure 5. Cumulative distribution functions created by averaging data deciles over participants and conditions, and similarly averaged deciles produced by ex-Gaussian (EXG) and EZ2 fits.

( $<1 \%$ ), estimates of Ter were too small to be plausible $(<100 \mathrm{msec})$. In such cases, we obtained parameter estimates by solving the EZ2 equations under the constraint that Ter $>100 \mathrm{msec}$. Note that without constraint, EZ2 parameters produce a perfectly accurate account of accuracy and correct RT mean and variance. Although this is not necessarily the case when a constraint is imposed, the effect of the constraint used on our data was negligible, so that the account of these measures remained essentially perfect.

Since the ex-Gaussian usually provides an excellent descriptive account of RT distribution, our methods also provided a gold standard against which to compare the diffusion model's account of the data, thus addressing concerns raised by Ratcliff (2008) about EZ estimation. A qualitative check provided by inspecting Figure 5 shows that for our data, EZ2 estimation produced an accurate account of the full distribution of correct RT, which was only slightly inferior to that of the ex-Gaussian. A small disadvantage is to be expected, given that the diffusion model accounts for accuracy, as well as RT, using the same number of parameters as the ex-Gaussian, which accounts only for RT.

\section{Results}

EZ2 parameter estimates were derived for each of the 23 participants from Experiment 1. Mean RT, RT variance, and error rate were used to estimate the nondecision time, evidence criterion, and drift rate parameters at each level of task and trial type. These parameter estimates were analyzed using a 3 (task: letter, digit, color) $\times 5$ (trial type: repeat, switch-to, switch-away, noninformative repeat, and noninformative switch) repeated measures ANOVA, followed by five simple comparisons for trial with familywise error rate adjusted at $\alpha=.01$. As well as the drift rate, criterion, and nondecision time parameters, we analyzed decision time. We will present results for all four measures for clarity, but it is important to keep in mind that these measures are related, since decision time is a function of drift rate and criterion, and decision time and nondecision time sum to mean RT. Task did not interact with trial type in any of these analyses, so we will report results averaged over task (Figure 6). As in earlier analyses, five planned contrasts compared repeat trials with switch-to, switchaway, and noninformative repeat trials and switch-away trials with switch-to and noninformative switch trials. We also will report correlations between EZ2 parameters and the early cue-locked switch positivity.

Figure 6A shows that nondecision time varied from $370 \mathrm{msec}$ for repeat trials to $650 \mathrm{msec}$ for noninformative switch trials [trial, $F(4,88)=71.06, p<.001, \varepsilon=.673$ ] Nondecision time was significantly shorter for repeat trials than for switch-away and noninformative repeat trials $[F(1,22)=71.41, p<.001$, and $F(1,22)=59.69, p<$ .001 , respectively] [although not part of the planned set, note that the repeat and noninformative switch trials comparison was also highly significant; $F(1,22)=213.78$, $p<.001]$. Nondecision time did not differ between repeat trials and switch-to trials $(F<1.5)$, but switch-away trials had a significantly shorter nondecision time, as compared with noninformative switch trials $[F(1,22)=28.49, p<$ $.001]$ and a longer nondecision time as compared with switch-to trials $[F(1,22)=112.8, p<.001]$. Larger cuelocked positivity was associated with shorter nondecision time for switch-to cues $(r=-.397, p<.05)$ and marginally for switch-away cues $(r=-.349, p=.051)$.

As is shown in Figure 6D, response criteria were low on repeat and both types of noninformative cue trials. However, criteria were significantly higher for both switch-to and switch-away trials [trial, $F(4,88)=14.74, p<.001$, $\varepsilon=.465$; repeat vs. switch-to, $F(1,22)=14.07, p=.001$; repeat vs. switch-away, $F(1,22)=9.73, p=.005]$. Decision time was also significantly affected by trial type $[F(4,88)=11.71, p=.001, \varepsilon=.341$; see Figure 6B] . Both switch-to and switch-away trials had significantly longer decision time than did repeat trials $[F(1,22)=$ $18.32, p<.001$, and $F(1,22)=9.14, p=.006$, respectively]. Decision time was also lower for noninformative switch than for switch-away trials $[F(1,22)=16.76, p<$ $.001]$. This can be accounted for by differences in response criterion $[F(1,22)=44.06, p<.001]$, but not drift rate $(F<1$; see Figure 6C). Drift rate for repeat trials was significantly higher than that for all other trial types [switchto, $F(1,22)=62.05, p<.001$; switch-away, $F(1,22)=$ $27.67, p<.001$; noninformative repeat, $F(1,22)=13.36$, $p=.001]$. Larger cue-locked positivity on switch-to trials was associated with a shorter decision time $(r=-.414$, $p<.05)$, lower criterion $(r=-.425, p<.05)$ and a faster drift rate $(r=.366, p<.05)$. Switch-away cues showed no significant correlations between cue-locked positivity and these diffusion measures.

\section{Discussion}

The nondecision time findings are consistent with predictions based on our assumption that the cues preceding noninformative switch, switch-away, and switch-to trials result in differential degrees of activation of an anticipatory reconfiguration process. Partially informative switchaway cues, which provided certainty about an upcoming task switch without indicating which task would be active, offered a reliable behavioral advantage over noninformative cues that were equally likely to be followed by a switch or a repeat trial. In particular, this advantage was 
A Nondecision Time (msec)

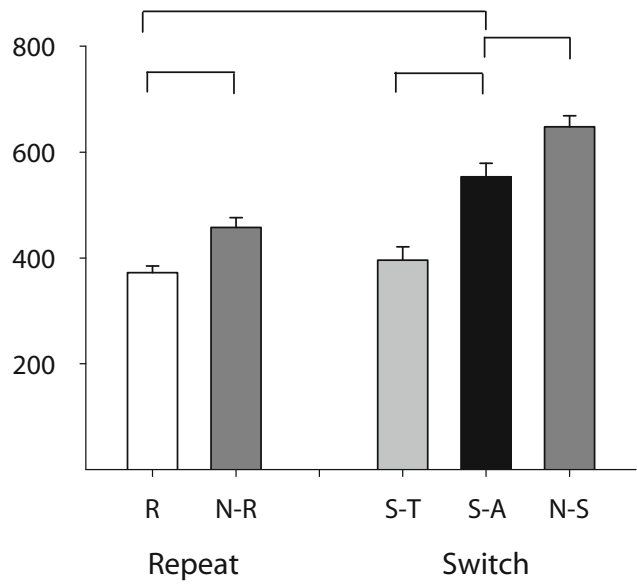

C

Drift Rate

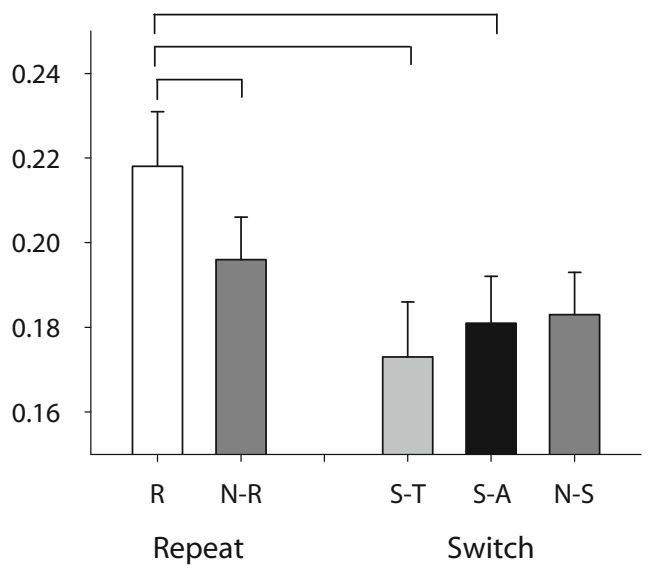

B Decision Time (msec)

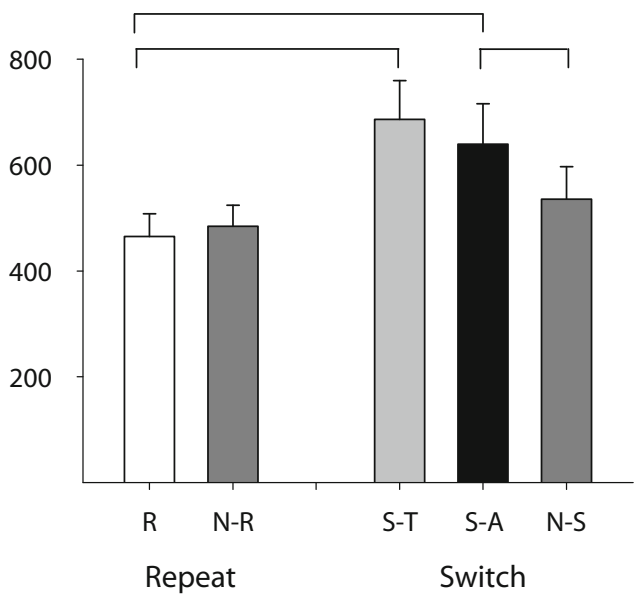

D

Criterion

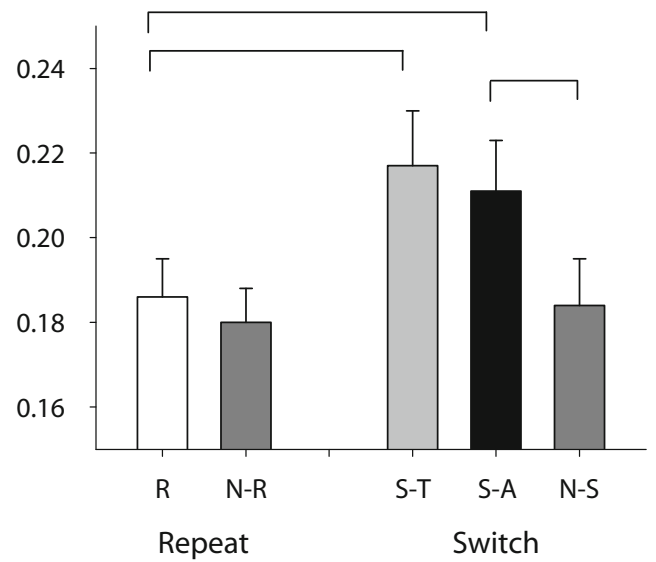

Figure 6. Diffusion model parameters. R, repeat; N-R, noninformative repeat; S-T, switch-to; S-A, switch-away; $\mathrm{N}-\mathrm{S}$, noninformative switch. Significant differences between conditions are shown by solid lines at $p<.01$.

evident in nondecision time, ${ }^{1}$ a latent measure that, in the context of cued task-switching, is affected by the degree of anticipatory reconfiguration afforded by the cue. In the present paradigm, the only common information provided by switch-to and switch-away cues and not afforded by noninformative cues is that the task that was relevant on the previous trial will not be repeated. The finding that this information resulted in a reduction in nondecision time suggests that both switch-away and switch-to cues elicit some degree of anticipatory reconfiguration and that this partial preparation results in a behavioral advantage over noninformative cues that are equally likely to result in a switch or repeat trial.

Just as is predicted by our speed-accuracy trade-off account, response criterion adjustment occurred only for cues validly predicting a change in task (switch-to, switchaway), but not for cues signaling that the task may repeat (noninformative). ${ }^{2}$ This criterion adjustment caused decision time to be greater in the switch-to and switch-away conditions than in the noninformative switch condition. The decision time difference between switch-away and noninformative cues masked the nondecision time advantage that partial preparation afforded to switch-away cues over noninformative switch cues, resulting in no observable difference in mean RT.

This pattern of reduced nondecision time and increased response criterion in the switch-away and switch-to conditions may appear counterintuitive. It suggests that anticipatory reconfiguration (reflected in reduced nondecision time) resulted in longer rather than shorter decision times - a disadvantage, rather than an advantage, of preparation. However, seeing this effect as only a disadvantage fails to appreciate the full range of behavior displayed by participants, and the task demands that they must satisfy in terms of accuracy, as well as speed. The increase in response criterion had the advantage of decreasing the probability of an error, which explains why accuracy was higher in the switch-away and switch-to conditions 
than in the noninformative switch condition, even though the quality of the evidence (drift rate) was the same in all three conditions. By setting the criterion as they did, participants were able to achieve greater accuracy in the switch-to condition without sacrificing speed, relative to the noninformative conditions, since the increased decision time cost was canceled by the nondecision time advantage afforded by partial preparation.

\section{GENERAL DISCUSSION}

Nicholson, Karayanidis, Davies, and Michie (2006) reported an early cue-locked differential switch positivity for both fully informative (switch-to) and partially informative (switch-away) cues, suggesting a common anticipatory reconfiguration process. They argued that since the only common information provided by these cues was that the previously active task would not be repeated, this switch positivity could reflect suppression or disengagement of the now irrelevant task set. However, the absence of a demonstrated behavioral benefit afforded by switch-away cues and the fact that both switch-to and switch-away, but not repeat, cues involved a change in spatial position suggested another interpretation: that the early switch positivity reflects processing of the change in the spatial position of the cue or repetition priming for the repeat cue. In the present study, we tested this alternative explanation by including noninformative cues that, like switch-to and switch-away cues, involve a change in spatial position (and therefore do not involve cue identity repetition) but, unlike switch-to and switch-away cues, are not associated with any strategic benefit in suppressing the previously active task set. The ERP data showed that the posterior cue-locked D-Pos1 was elicited for both switch-to and switch-away cues, but not for noninformative cues. Therefore, D-Pos 1 does not simply reflect processing of a change in cue position.

These results indicate that partially informative cues trigger a subcomponent of an anticipatory reconfiguration process represented by the D-Pos1 to both switchto and switch-away cues. Surprisingly, switch-away cues signaling that the upcoming trial required a change in task set, without specific information about which task set to prepare, did not appear to provide any advantage in speed, relative to noninformative cues signaling that a change might or might not be necessary. However, error scores provided evidence that the failure to find a switchto advantage in mean RT was due to a speed-accuracy trade-off.

We examined the issue of speed-accuracy trade-off by using the EZ2 analysis method (Grasman et al., 2009), which combines measurements of response accuracy with measurements of response speed and variability in order to fit an evidence accumulation model of the task decision process. Critically for our purposes, this model produces estimates of the criterion amount of evidence required to make a decision and of the mean time to complete nondecision and decision processes. Diffusion model analyses provided evidence of a behavioral effect on RT of the partial information provided by switch-away cues. Specifi- cally, nondecision time, a latent measure that includes the time to complete reconfiguration after target onset, did not differ between repeat and switch-to cues but increased progressively across switch-to, switch-away, and noninformative cues. Hence, cues that allowed full reconfiguration showed no effect of reconfiguration on nondecision time, whereas cues that allowed partial reconfiguration provided a nondecision time advantage over cues providing no information about the likelihood of a switch trial.

These results are consistent with the idea that the partial preparation afforded by the information that the previously active task set will not be repeated is a timeconsuming part of the reconfiguration process. Nondecision time was negatively correlated with the amplitude of the early cue positivity, suggesting that activation of the processes reflected in this early switch positivity resulted in greater anticipatory reconfiguration. Importantly, the diffusion analysis demonstrates that behavioral results are consistent with the interpretation of D-Pos1 as being representative of preparation for an upcoming change in task set. These data provide a crucial link between behavioral and ERP data that does not exist when only mean RT is considered.

Although these findings strongly support the contention that partially informative cues trigger some anticipatory reconfiguration process, there are at least two possible interpretations of the precise nature of this process. One possibility is inhibition of the previously relevant task set, which both switch-to and switch-away cues indicate will not be relevant on the current trial (Nicholson, Karayanidis, Davies, \& Michie, 2006). Another is activation of one or more task sets that the cues indicate are likely to be relevant for the following target. ${ }^{3}$ In the latter case, switch-away cues could either activate both possible task sets or randomly activate one of the two possible task sets. If both possible task sets are activated, it seems likely that cue-locked waveforms would reflect greater processing for switch-away trials than for switch-to trials. Hence, cue-locked differentiation between repeat and switch trials (i.e., D-Pos1) should be larger or more prolonged for switch-away cues than for switch-to cues (i.e., switch-to trials $=$ one task set activation, switch-away trials $=$ two task set activations). Furthermore, noninformative cues are also likely to activate the nonrepeat task set. Therefore, the cue-locked positivity should show amplitude changes so that repeat $<$ switch-to $=$ noninformative $<$ switchaway. This order is not compatible with the pattern of differences observed in cue-locked waveforms.

If switch-away cues activate only one of the two cued task sets in a random or semirandom fashion, the behavioral advantages that we found for switch-away over noninformative cues are difficult to understand. Since both types of cues afford the same level of uncertainty reduction about the nature of the upcoming task, it seems likely that both will be used to activate the corresponding task sets in the same way. If this were the case, there should be no behavioral advantage for switch-away trials over noninformative trials, which is not what was observed. It remains possible, however, that the task set activation account is correct if participants only, or more efficiently, 
use switch-away cues for task set activation, although it is unclear why this might be the case.

The alternative interpretation (Nicholson, Karayanidis, Davies, \& Michie, 2006) is that anticipatory task set reconfiguration is a multicomponent process that encompasses both inhibition of the previously active task set, reflected in the early D-Pos1, and activation of the now relevant task set, reflected in the later D-Pos2. Variation across switch-to, switch-away, and noninformative cues in both D-Pos 1 and nondecision time is compatible with a process of suppression or inhibition of the previously active task set, which may be conceptualized as being similar to the idea of disengagement of attention to spatial location invoked in cued spatial attention tasks (e.g., Posner, 1980; but see Cohen, Romero, Servan-Schreiber, \& Farah, 1994). This interpretation is strengthened by the finding that the amplitude of the early cue-locked positivity for both switch-to and switch-away cues was inversely related to mean RT and nondecision time, suggesting that greater anticipatory reconfiguration, which we argue involves inhibition of the irrelevant task set, leads to a shorter RT by reducing nondecision time.

The evidence accumulation (diffusion) model analysis provided not only evidence for a behavioral benefit arising as a result of task set inhibition, but also a plausible explanation of why this behavioral benefit is not evident in mean RT measures. Specifically, model parameters indicated that the nondecision time advantage offered by this partial preparation was not evident in mean RT because it was counteracted by another process that was also activated by cues that provided certainty of an upcoming switch in task, which resulted in an increase in the decision time component of RT. Estimates of the criterion amount of evidence required to make a decision indicated that the participants responded to cues that provided certainty of an upcoming switch in task (i.e., switch-away and switchto cues) by requiring a higher standard of evidence, resulting in slower but more accurate decisions for switch-away than for noninformative switch trials.

This more fine-grained analysis of the behavioral data produced results that, in contrast to traditional approaches, are able to provide a unified explanation of both accuracy and speed. The fact that switch-to and switch-away cues were associated with both a reduction in nondecision time and an increase in evidence criterion suggests a third interpretation of the anticipatory preparation process reflected in the early cue-locked positivity. Specifically, it is possible that D-Pos1 reflects the process of increasing the evidence criterion and that this is a time-consuming process that contributes to nondecision time. When this process can be completed before target onset, D-Pos 1 is elicited in the cue-target interval, and nondecision time is reduced. When it is completed after target onset, D-Pos 1 is elicited after target onset, and nondecision time is higher. Although this explanation is compatible with most of our results, it predicts that evidence criterion should be higher for all switch trials, but this was not the case for noninformative switch trials. This account is also not easily reconciled with the fact the target-locked positivity was elicited for both noninformative switch and noninformative repeat trials, even though neither showed an increase in evidence criterion. Furthermore, it predicts that the amplitude of the early cue positivity will be associated with a higher evidence criterion for both switch-to and switch-away cues. However, a larger early cue positivity was associated with a shorter nondecision time and a lower evidence criterion, the latter being significant only for switch-to cues.

In conclusion, we have replicated evidence for an early cue-locked positivity that is elicited by cues that provide certainty of an upcoming switch in task. We provided strong evidence that this positivity is associated with an anticipatory component of the task set reconfiguration process and with a behavioral benefit in the nondecision component of RT. We have identified a number of alternative interpretations of this process and have shown that most fail to explain the full set of behavioral and ERP data. It seems to us arguable, therefore, that although the data do not provide direct evidence for task set inhibition as a component of anticipatory task set reconfiguration, this interpretation provides the most plausible and comprehensive account of the data.

More broadly, the finding that simple behavioral measures and ERP measures may lead to theoretically opposed interpretations of the underlying cognitive processes suggests that such simple behavioral measures alone may be limited. We argue, instead, that more sophisticated modelbased analyses of behavior, combined with ERP and other neuroimaging measures, are likely to be more successful in providing a full account of all relevant processes (see also Forstmann et al., 2008).

\section{AUTHOR NOTE}

This work was supported by funding from the University of Newcastle Research Grants Committee and was approved by the University of Newcastle Human Research Ethics Committee. Elise Mansfield was supported by an Australian Postgraduate Award and a University of Newcastle Vice-Chancellor's Award for Outstanding RHD Candidate. We acknowledge the contribution of Rebecca Nicholson and Damien Mannion in setting up the paradigm. We thank Pat Michie for discussions related to this work. Correspondence concerning this article should be addressed to F. Karayanidis, Functional Neuroimaging Laboratory, School of Psychology, University of Newcastle, Callaghan, NSW 2308, Australia (e-mail: frini.karayanidis@newcastle.edu.au).

\section{REFERENCES}

Andreassi, J. L. (2000). Psychophysiology: Human behavior and physiological response (4th ed.). Mahwah, NJ: Erlbaum.

Arrington, C. M., Logan, G. D., \& Schneider, D. W. (2007). Separating cue encoding from target processing in the explicit task-cuing procedure: Are there "true" task switch effects? Journal of Experimental Psychology: Learning, Memory, \& Cognition, 33, 484-502. doi:10.1037/0278-7393.33.3.484

Cohen, J. D., Romero, R. D., Servan-Schreiber, D., \& Farah, M. J. (1994). Mechanisms of spatial attention: The relation of macrostructure to microstructure in parietal neglect. Journal of Cognitive Neuroscience, 6, 377-387. doi:10.1162/jocn.1994.6.4.377

Driesbach, G., Haider, H., \& Kluwe, R. H. (2002). Preparatory processes in the task-switching paradigm: Evidence from the use of probability cues. Journal of Experimental Psychology: Learning, Memory, \& Cognition, 28, 468-483. doi:10.1037/0278-7393.28.3.468

Forstmann, B. U., Dutilh, G., Brown, S., Neumann, J., von Cramon, D. Y., Ridderinkhof, K. R., \& WagenmaKers, E.-J. (2008). Striatum and pre-SMA facilitate decision-making under time pressure. Proceedings of the National Academy of Sciences, 105, 1753817542. 
Grasman, R. P. P. P., Wagenmakers, E.-J., \& van der Maas, H. L. J. (2009). On the mean and variance of response times under the diffusion model with an application to parameter estimation. Journal of Mathematical Psychology, 53, 55-68. doi:10.1016/j.jmp.2009.01.006

Heathcote, A., Brown, S., \& Mewhort, D. J. K. (2002). Quantile maximum likelihood estimation of response time distributions. Psychonomic Bulletin \& Review, 9, 394-401.

Hübner, M., Dreisbach, G., Haider, H., \& KLuwe, R. H. (2003). Backward inhibition as a means of sequential task-set control: Evidence for reduction of task competition. Journal of Experimental Psychology: Learning, Memory, \& Cognition, 29, 289-297. doi:10.1037/0278 $-7393.29 .2 .289$

Ille, N., Berg, P., \& Scherg, M. (2002). Artifact correction of the ongoing EEG using spatial filters based on artifact and brain signal topographies. Journal of Clinical Neurophysiology, 19, 113-124.

Karayanidis, F., Coltheart, M., Michie, P. T., \& Murphy, K. (2003). Electrophysiological correlates of anticipatory and posttarget components of task-switching. Psychophysiology, 40, 329-348. doi:10.1111/1469-8986.00037

Kieffaber, P. D., \& Hetrick, W. P. (2005). Event-related potential correlates of task switching and switch costs. Psychophysiology, 42, 5671. doi:10.1111/j.1469-8986.2005.00262.x

Koch, I., \& Philipp, A. M. (2005). Effects of response selection on the task repetition benefit in task switching. Memory \& Cognition, 33, 624-634.

LucE, R. D. (1986). Response times. New York: Oxford University Press.

Mayr, U., \& Keele, S. W. (2000). Changing internal constraints on action: The role of backward inhibition. Journal of Experimental Psychology: General, 129, 4-26. doi:10.1037/0096-3445.129.1.4

Meiran, N., Chorev, Z., \& SaPir, A. (2000). Component processes in task switching. Cognitive Psychology, 41, 211-253. doi:10.1006/ cogp. 2000.0736

Miniussi, C., Marzi, C. A., \& Nobre, A. C. (2005). Modulation of brain activity by selective task sets observed using event-related potentials. Neuropsychologia, 43, 1514-1528. doi:10.1016/j .neuropsychologia.2004.12.014

Nicholson, R., Karayanidis, F., Bumak, E., Poboka, D., \& Michie, P. (2006). ERPs dissociate the effects of switching task sets and task cues. Brain Research, 1095, 107-123. doi:10.1016/j.brainres.2006.04.016

Nicholson, R., Karayanidis, F., Davies, A., \& Michie, P. T. (2006). Components of task set reconfiguration: Differential effects of "switch-to" and "switch-away" cues. Brain Research, 1121, 160-176. doi:10.1016/j.brainres.2006.08.101

Nicholson, R., Karayanidis, F., Рoboka, D., Heathcote, A., \& Michie, P. T. (2005). Electrophysiological correlates of anticipatory task-switching processes. Psychophysiology, 42, 540-554. doi:10.1111/j.1469-8986.2005.00350.x

Posner, M. I. (1980). Orienting of attention. Quarterly Journal of Experimental Psychology, 32, 3-25. doi:10.1080/00335558008248231
Ratcliff, R. (2008). The EZ diffusion method: Too EZ? Psychonomic Bulletin \& Review, 15, 1218-1228.

Rogers, R. D., \& Monsell, S. (1995). Costs of a predictable switch between simple cognitive tasks. Journal of Experimental Psychology: General, 124, 207-231. doi:10.1037/0096-3445.124.2.207

Rushworth, M. F., Passingham, R. E., \& Nobre, A. C. (2005). Components of attentional set-switching. Experimental Psychology, 52, 83-98. doi:10.1027/1618-3169.52.2.83

SCHUCH, S., \& KoCH, I. (2003). The role of response selection for inhibition of task sets in task shifting. Journal of Experimental Psychology: Human Perception \& Performance, 29, 92-105. doi:10.1037/0096 $-1523.29 .1 .92$

SnOdgrass, J. G., \& Corwin, J. (1988). Pragmatics of measuring recognition memory: Applications to dementia and amnesia. Journal of Experimental Psychology: General, 117, 35-50. doi:10.1037/0096 $-3445.117 .1 .34$

VASEY, M., \& THAYER, J. (1987). The continuing problem of false positives in repeated measures ANOVA in psychophysiology: A multivariate solution. Psychophysiology, 24, 479-486. doi:10.1111/j.1469-8986.1987 .tb00324.x

WagenMakers, E.-J., van der MaAs, H. L. J., Dolan, C. V., \& GrasMAN, R. P. P. P. (2008). EZ does it! Extensions of the EZ-diffusion model. Psychonomic Bulletin \& Review, 15, 1229-1235.

WAgenMaKers, E.-J., VAN Der MaAs, H. L. J., \& Grasman, R. P. P. P. (2007). An EZ-diffusion model for response time and accuracy. Psychonomic Bulletin \& Review, 14, 3-22.

Wylie, G. R., \& Allport, D. A. (2000). Task switching and the measurement of "switch costs." Psychological Research, 63, 212-233. doi:10.1007/s004269900003

\section{NOTES}

1. For readers concerned that this behavioral effect is entirely dependent on the diffusion model's being correct, it is important to note that differences in Ter between conditions equal differences in the shorter RTs for those conditions. Hence, an interpretation of these results purely in terms of observed behavior is that switch-away cues reliably speed up the fastest responses, relative to noninformative cues.

2. As with nondecision time effects, criterion differences correspond to an observable behavioral difference. In the case of criterion effects, this is RT variance. When drift rate (which also affects RT variance) is the same between two conditions (e.g., switch-to and noninformative in our data) but one condition has a larger criterion (e.g., switch-to has a greater criterion than does noninformative in our data), it will also have a larger variance.

3. We thank an anonymous reviewer for this suggestion.

(Manuscript received May 29, 2008; revision accepted for publication February 8, 2009.) 